\title{
Dynamic Soil-Structure Analysis of Tower-Like Structures Using Spectral Elements
}

\author{
Davorin PENAVA, Ivan KRAUS, Mira PETRONIJEVIĆ, Günther SCHMID
}

\begin{abstract}
In this paper general equation system for linear dynamic soil-structure interaction (SSI) in frequency domain is presented. The main objective of the paper is to provide and investigate a possibility to use spectral elements in SSI domain. Spectral elements reduce considerably number of unknowns and in some cases, e.g. in frame structures coupled with analytically obtained impedance functions of sub-grade, produce no modelling errors. Results for two different shallow-founded beam structures with identical foundations excited by harmonic free-field motions are presented.
\end{abstract}

Keywords: frequency domain; impedance functions; shallow founded tower-like structures; soil-structure interaction; spectral elements

\section{INTRODUCTION}

Earthquake is a frequency rich signal that affects soilfoundation-structure system and thus introduces interaction between them. As described in EN 1998-5 [1] and EN 1998-6 [2], for tower-like structures, soilstructure effects should be accounted for elongation of the fundamental period, increasing of overall damping due to radiation and increasing of base rocking due to total displacement (soil and structure deformation combined with foundation rotation).

Numerical methods for solving SSI problems are commonly divided into two categories: i) direct method in time domain and ii) substructure method in frequency domain. Beside these two methods, hybrid method can also be introduced. The direct method incorporates the whole soil-foundation-structure system and allows nonlinear analysis, which is, on the other hand, not possible to conduct in frequency domain. Nevertheless, the time-domain solutions can be extremely resource- and time-demanding.

This paper presents the advantages of the frequency domain approach where the basic SSI equations are formulated in total displacements using the spectral element method (SEM), suitable for engineers due to similitude to finite element technique. The effective seismic forces are proportional to a prescribed base rock motion or to the equivalent free field motion of an earthquake. It is obvious that the seismic forces affect both the structure and the sub-grade. Waves propagate through soil and affect the structure, where they continue to propagate through the structural members. These waves are refracted and reflected at the junctions or at the ends of members and finally constitute the entire dynamic response. Usually, we calculate this dynamic response in time or frequency domain using finite element (FE) techniques in direct or sub-structure approach. The accuracy of the results depends on the modelling of the structure and the soil.

A special advantage occurs if the frame structure is modelled through spectral elements (SE). The frequency domain formulation of the element dynamic soil-stiffness matrix enables direct coupling with the impedance matrix of the soil, which is also frequency dependent and for different kinds of foundation accessible in the literature [3]. The basic equations are analogues to those in the finite element method with which the structural engineers are familiar. Also, the usage of spectral elements reduces the number of unknowns and increases the accuracy of the numerical results. The analysis in the frequency domain can be extended to non-linear dynamic behaviour, which has been shown in [4] and [5].

The dynamic stiffness matrix is obtained from the exact analytical solution of the governing differential equation of motion of the beam element undergoing free natural vibration. For this reason, the dynamic stiffness matrix is often called exact. The dynamic stiffness matrix of a Bernoulli-Euler beam element was derived by Kolousek in 1941 [6]. After that he developed the dynamic stiffness matrices with the effects of shear deformation and rotational inertia (see [7] and [8]) while Banerjee [9] derived the dynamic stiffness matrix for an axially loaded bending-torsion coupled beam element. Further investigations resulted in the dynamic stiffness matrix of a cracked Timoshenko beam (see [10] and [11]). Doyle was the first who introduced the name spectral element [12].

There are only a few applications of SEM to investigate vibration of the structures [13]. In most cases the structure consists of one single beam [10] or a simple truss [14] subjected to a dynamic force. Penava applied SEM to calculate response of $2 \mathrm{D}$ frame structure subjected to a Rayleigh wave ([15-17]). Petronijevic et al. used this approach to analyse the influence of traffic induced vibration in buildings [18]. Also, Hilmer [4] had demonstrated the applicability of the SEM and Laplace transform to the non-linear dynamic SSI problem. The purpose of this paper is to formulate SSI in a compact and concise way using SEM and to show the applicability of spectral and frequency domain methods to analysis of earthquake excited structures.

\section{SYSTEM EQUATION}

Dynamic soil-structure interaction problems can be analysed in time or frequency domain. If the frequency domain is considered, a structure can be modelled by using spectral elements while the soil dynamic stiffness matrices can be modelled using the most suitable numerical method, the boundary element method [19], the thin layer method [20] or an analytical method [21]. Using the master-slave concept dynamic stiffness 
matrices, which are complex, frequency dependent and introduce stiffness, damping and mass properties, can be developed considering only the essential DOF's without loss of accuracy. Any linear rheological material behaviour can be modelled with frequency dependent complex material functions. By coupling the substructures, using the direct stiffness method [8], the discrete equation of the soil-structure system in the total displacements formulation (elastic deformation plus rigid body motion) is obtained as:

$$
\left[\begin{array}{ccc}
K_{S S}^{S} & K_{S I}^{S} & 0 \\
K_{I S}^{S} & K_{I I}^{S}+K_{I I}^{F} & K_{I G}^{F} \\
0 & K_{G I}^{F} & K_{G G}^{F}
\end{array}\right]\left[\begin{array}{c}
V_{S} \\
V_{I} \\
V_{G}
\end{array}\right]=\left[\begin{array}{c}
P_{S} \\
P_{I} \\
P_{G}
\end{array}\right]
$$

where $K^{S}$ and $K^{F}$ are the dynamic stiffness matrices of the sub-structures (exponent $S$ stands for structure while $F$ stands for foundation or soil).

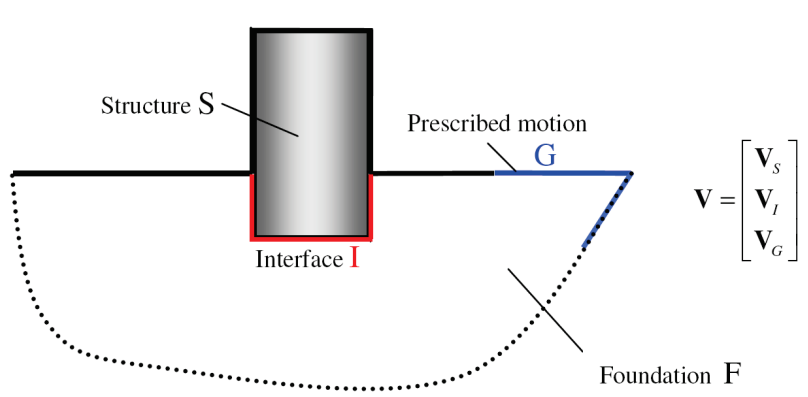

Figure 1 Definition of kinematic DOF's

The system's N kinematic DOF's $V$ are partitioned in those on the interface $V_{\mathrm{I}}$, structure $V_{\mathrm{S}}$ and the sub-grade $V_{G}$. In those DOF's internal forces may act. $P_{S}, P_{I}$ and $P_{G}$ are the dynamic forces that correspond to the kinematic DOF's as indicated in Fig. 1. They are complex and frequency dependent. We assume that the seismic event is introduced at the boundary $G$ of the considered region. Also we assume that this event is described as a specified time-harmonic ground motion $V_{G} e^{i \omega t}$ with frequency $\omega$. No other external loads are assumed to act on the structure, that is $P_{S}=P_{I}=0$. Then the unknown structural displacements $V_{\mathrm{S}}$ and $V_{\mathrm{G}}$ are obtained from the first two equations of the matrix in Eq. (1) resulting with:

$$
\left[\begin{array}{cc}
K_{S S}^{S} & K_{S I}^{S} \\
K_{I S}^{S} & K_{I I}^{S}+K_{I I}^{F}
\end{array}\right]\left[\begin{array}{c}
V_{S} \\
V_{I}
\end{array}\right]=\left[\begin{array}{c}
P_{S} \\
-K_{I G}^{F} V_{G}
\end{array}\right]
$$

In Eq. (2) the term $-K_{I G}^{F} V_{G}=P_{\text {eff, } I}$ is the driving force of the motion acting at the interface $I$. The seismic source is usually far away so that it cannot be included within the model. Then as an alternative description of the seismic event the free-field motion $V_{I}^{\prime}$ may be used. The relation between the free-field motion $V_{I}^{\prime}$ and prescribed motion $V_{G}$ can be obtained when terms of the structure are omitted from Eq. (2). Thus equation

$$
K_{I I}^{F} V_{I}^{\prime}=-K_{I G}^{F} V_{G}
$$

defines the free-field motion $V_{I}{ }^{\prime}=-\left(K_{I I}^{F}\right)^{-1} K_{I G}^{F} V_{G}$ in terms of prescribed motion $V_{G}$ and, at the same time, the driving force

$$
P_{e f f, I}=K_{I I}^{F} V_{I}^{\prime}=-K_{I G}^{F} V_{G}
$$

acting at the interface $I$. Thus, the final system of equations can be written together with Eq. (4) as:

$$
\left[\begin{array}{cc}
K_{S S}^{S} & K_{S I}^{S} \\
K_{I S}^{S} & K_{I I}^{S}+K_{I I}^{F}
\end{array}\right]\left[\begin{array}{c}
V_{S} \\
V_{I}
\end{array}\right]=\left[\begin{array}{c}
0 \\
P_{e f f, I}
\end{array}\right]
$$

If a rigid base plate, with mass $M$ and mass moment of inertia with respect to its centre of gravity $I_{\mathrm{r}}$ is considered in the analysis, its impedance is added to $K_{I I}^{S}$. In this case Eq. (5) results in

$$
\left[\begin{array}{cc}
K_{S S}^{S} & K_{S I}^{S} \\
K_{I S}^{S} & K_{I I}^{S}+K_{I I}^{F}-\omega M^{F}
\end{array}\right]\left[\begin{array}{c}
V_{S} \\
V_{I}
\end{array}\right]=\left[\begin{array}{c}
0 \\
P_{e f f, I}
\end{array}\right]
$$

where $M^{F}$ is a diagonal sub-matrix with entries $M$ and $I_{\mathrm{r}}$ for translational and rotational DOFs respectively. Recent study [22] showed that a response of a soil-foundationstructure system is the same whether the foundation mass is included or not. The results, obtained through numerical analyses, were compared with results of experiments carried out in a geotechnical centrifuge, as well as on a shaking table. The assumption on massless foundation was used in earlier studies ([23-26]), also. Nevertheless, it is clear from Eq. (6) that for slender structures with heavy foundations, foundation mass may influence its response.

\section{SPECTRAL ELEMENT METHOD}

To improve the accuracy in the FE analysis at high frequency excitation it is necessary to use an extremely fine FE mesh, which may increase computational cost and time. Such a problem can be resolved by using spectral element method (SEM). SEM is a well-known approach explained in detail by Doyle [12]. The approach has been known from 1941 when Kolousek derived the dynamic stiffness matrix for the first time. In the last 40 years numerous dynamic stiffness matrices for beam and plate elements have been developed (e.g. [12], [27]). Spectral beam elements, with constant material and geometrical properties (see Fig. 2) provide exact solutions due to the fact that their interpolation functions satisfy the governing differential equations of the element undergoing free vibration:

$$
\begin{aligned}
& E A u^{\prime \prime}-\omega^{2} \bar{m} u=0 \\
& E I v^{(4)}-\omega^{2} \bar{m} v=0
\end{aligned}
$$

where $E, A, I$ and $\bar{m}$ are the Young's modulus, area of cross section, moment of inertia and the mass per unit length of the beam respectively. The wave Eqs. (7) in the frequency domain for axial deformation and for bending 
are ordinary differential equations of $2^{\text {nd }}$ and $4^{\text {th }}$ order respectively. In Eq. (7) $u$ and $v$ stand for complex spectral amplitudes of displacements along the beam in

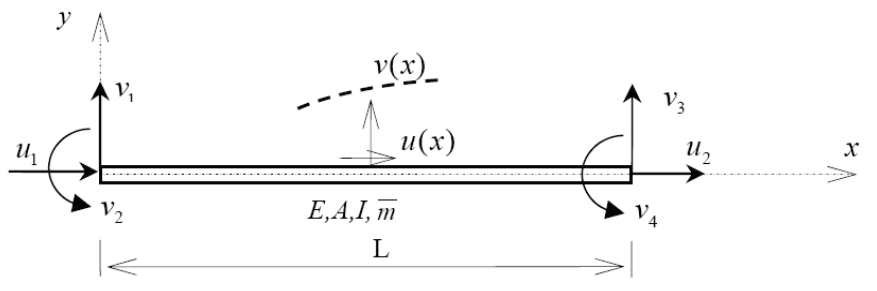

Figure 2 Euler-Bernoulli beam spectral element
The solutions for Eqs. (7) are obtained as linear combination of independent exponential functions $e^{i k j x}$ :

$$
\begin{aligned}
& u(x)=\sum_{j=1}^{2} \alpha_{j} e^{i k_{j} x} \\
& v(x)=\sum_{j=1}^{4} \beta_{j} e^{i k_{j} x}
\end{aligned}
$$

where $k_{j}$ is the wave number, $x$ is the direction along the beam and $i^{2}=1$. Wave numbers $k_{j}$ are the characteristic roots of the equations obtained by inserting the exponential functions from Eq. (8) into the wave Eq. (7). They depend on properties of the beam and frequency $\omega$. Integration constants $\alpha$ and $\beta$ can be expressed through the kinematic values at the beam's ends resulting in the stiffness matrices of the axial deformation of the beam, $K_{A}$, and bending, $K_{B}$, are obtained using the relations between the corresponding nodal displacements and forces. The exact values for the spectral stiffness elements can be found in literature $([12,27])$. As the SE matrix is derived in frequency domain for each frequency $\omega$, the general solution is represented by the combination of an frequency dependent dynamic shape functions. Dynamic longitudinal and transversal direction respectively (Fig. 2). infinite number of wave trains of different frequencies, i.e. spectral forms.

Deformations along the element in local coordinates for the frequency $\omega$ are obtained in the time domain through the relations:

$$
\begin{aligned}
& u(x, t)=\operatorname{Re}\left[\boldsymbol{N}_{A}(x, \omega) u e^{i \omega t}\right] \\
& v(x, t)=\operatorname{Re}\left[\boldsymbol{N}_{B}(x, \omega) v e^{i \omega t}\right]
\end{aligned}
$$

where $\boldsymbol{N}_{A}$ and $\boldsymbol{N}_{B}$ are matrices of interpolation functions provided and explained in Appendix at the end of this paper. Here structural damping is introduced through a complex elasticity modulus $E(1+i \eta)$ where $\eta$ is a frequency independent hysteretic damping coefficient, since the damping in the soil should be independent of frequency $([28-31])$.

\section{NUMERICAL EXAMPLES}

Two different structures, a chimney and an antenna, founded on the same type of shallow foundation are analyzed (see Fig. 3).

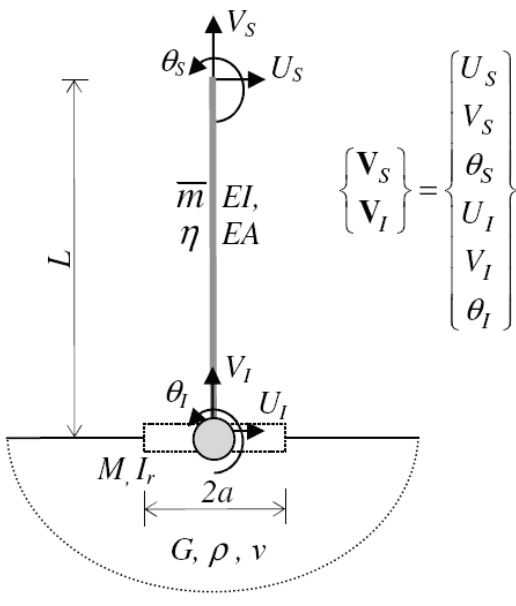

c)

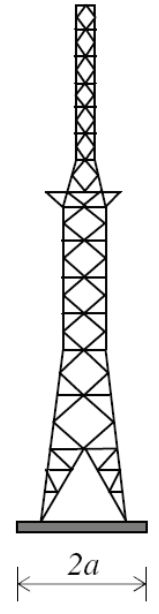

b)

Figure 3 Chimney (a), antenna (b) and model of soil-structure system (c)

Each structure is modelled through one spectral Bernoulli beam element with length $L$, cross section $A$, bending moment of inertia $I$, density $\rho$ and an elasticity modulus $E$, which is rigidly connected to a concrete square foundation plate with edge lengths of $2 a$ and thickness $t$. The mass of the foundation plate is $M$, its mass moment of inertia with respect to its centre of 
gravity is $I_{\mathrm{r}}$. The geometric and material characteristics of structures, foundation plate and soil are given in Tab. 1.

Table 1 Properties of sub-structures

\begin{tabular}{|c|c|c|}
\hline & Chimney & Antenna \\
\hline Parameters & Numerical values & Numerical values \\
\hline$E\left(\mathrm{~N} / \mathrm{m}^{2}\right)$ & $28 \times 10^{9}$ & $21 \times 10^{10}$ \\
\hline$\rho\left(\mathrm{kg} / \mathrm{m}^{3}\right)$ & 3500 & 7850 \\
\hline$L(\mathrm{~m})$ & 180 & 62 \\
\hline$A\left(\mathrm{~m}^{2}\right)$ & 22 & 0.075 \\
\hline$I\left(\mathrm{~m}^{4}\right)$ & 517 & 2.19 \\
\hline$\eta(-)$ & 0,10 & 0,10 \\
\hline & \multicolumn{2}{|c|}{ Foundation block } \\
\hline Parameters & \multicolumn{2}{|c|}{ Numerical values } \\
\hline$\rho\left(\mathrm{kg} / \mathrm{m}^{3}\right)$ & \multicolumn{2}{|c|}{2400} \\
\hline$M(\mathrm{t})$ & \multicolumn{2}{|c|}{810} \\
\hline$a(\mathrm{~m})$ & \multicolumn{2}{|c|}{7,5} \\
\hline$I_{\mathrm{r}}\left(\mathrm{tm}^{2}\right)$ & \multicolumn{2}{|c|}{3949} \\
\hline & \multicolumn{2}{|c|}{ Soil } \\
\hline Parameters & \multicolumn{2}{|c|}{ Numerical values } \\
\hline$G\left(\mathrm{~N} / \mathrm{m}^{2}\right)$ & \multicolumn{2}{|c|}{$24 \times 10^{6}$} \\
\hline$\rho\left(\mathrm{kg} / \mathrm{m}^{3}\right)$ & \multicolumn{2}{|c|}{1900} \\
\hline$v(-)$ & \multicolumn{2}{|c|}{0,4} \\
\hline
\end{tabular}

Impedance functions for a square massless rigid foundation on the elastic half-space with shear modulus $G$, density $\rho$ and Poisson's ratio $v$ are taken from [32] for the range of dimensionless frequencies $a_{0}=0$ to $a_{0}=2$ and then extrapolated to higher values. No material damping in the soil is considered. The impedance

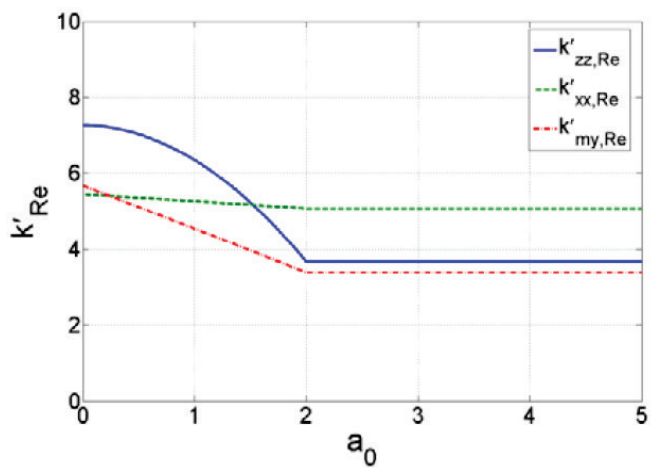

a) functions in horizontal (h), vertical (r) and rotational direction (r) are written respectively as

$$
\begin{aligned}
& K_{h}^{F}=G a\left[k_{x x, R e}^{\prime}\left(a_{0}\right)+i k_{x x, I m}^{\prime}\left(a_{0}\right)\right] \\
& K_{v}^{F}=G a\left[k_{z z, \operatorname{Re}}^{\prime}\left(a_{0}\right)+i{k_{z z, I m}^{\prime}}^{\prime}\left(a_{0}\right)\right] \\
& K_{r}^{F}=G a^{3}\left[k_{m y, \operatorname{Re}}^{\prime}\left(a_{0}\right)+i k_{m y, I m}^{\prime}\left(a_{0}\right)\right]
\end{aligned}
$$

where prime indicates non-dimensional impedance functions, whose real parts are $k_{x x, R e}^{\prime}, k_{z z, R e}^{\prime}$ and $k_{m y, R e}^{\prime}$, while imaginary parts are $k_{x x, I m}^{\prime}, k_{z z, I m}^{\prime}, k_{m y, I m}^{\prime}$. The nondimensional impedances are presented as functions of a dimensionless frequency $a_{0}$ in Fig. 4 . The dimensionless frequency is equal to

$$
a_{0}=\frac{a \omega}{\sqrt{\frac{G}{\rho}}}
$$

where $\omega$ is circular frequency and $a$ is the half-width of the foundation plate. The coupling terms between swaying and rocking are neglected in the case of surface foundation $[25,33,34]$.

For each frequency $\omega$ the motion of the mechanical model of the system (Fig. 3c) is represented with six DOF's at the beam's end.

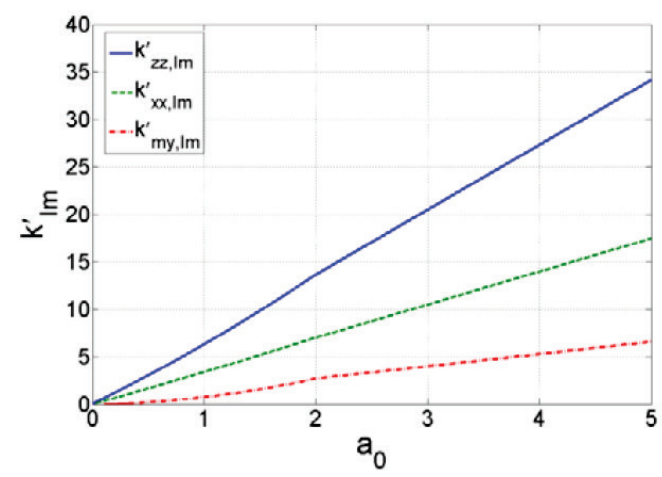

b)

Figure 4 Real (a) and imaginary part (b) of non-dimensional impedance functions

The seismic excitation is introduced with harmonic Rayleigh wave field. We assume that the length of the foundation is small compared to the length of a Rayleigh wave. Since the horizontal and vertical responses of the structure are decoupled we analyze the horizontal and the vertical motion separately. The horizontal and vertical component of the free field wave at the interface $I$ are chosen as a cosine-function with amplitude in horizontal and vertical directions $\left|U_{i}^{\prime}\right|$ and $\left|V_{i}^{\prime}\right|$ respectively. In all analyzed examples the material damping of the soil is set to zero in order to clearly show the influence of radiation damping on the structural response. First, the static stiffness values of the beam rigidly attached to the base:

$K_{h}^{S}=\frac{3 E I}{l^{3}}, K_{v}^{S}=\frac{E A}{l}$ and $K_{r}^{S}=\frac{3 E I}{l}$ are compared with the static stiffness values of the foundation:

$K_{h}^{F}=G a K_{x x, R e}^{\prime}, K_{v}^{F}=G a K_{z z, R e}^{\prime}$ and

$K_{r}^{F}=G a^{3} K_{m y, R e}^{\prime}$

which are calculated and provided in Tab. 2.

Table 2 Static system values of sub-systems

\begin{tabular}{lccc}
\hline \multicolumn{1}{c}{ Sub-structure } & $\begin{array}{c}K_{\mathrm{h}} \\
(\mathrm{N} / \mathrm{m})\end{array}$ & $\begin{array}{c}K_{\mathrm{v}} \\
(\mathrm{N} / \mathrm{m})\end{array}$ & $\begin{array}{c}K_{\mathrm{r}} \\
(\mathrm{Nm} / \mathrm{rad})\end{array}$ \\
\hline \hline Chimney & $7,45 \times 10^{6}$ & $3,42 \times 10^{9}$ & $241,0 \times 10^{9}$ \\
Antenna & $5,74 \times 10^{6}$ & $0,25 \times 10^{9}$ & $22,53 \times 10^{9}$ \\
Sub-grade & $1,31 \times 10^{9}$ & $0,98 \times 10^{9}$ & $57,41 \times 10^{9}$ \\
\hline
\end{tabular}

In case of the chimney, the static stiffness values of the structure for vertical and rotational motion are about 
3,5 and 4,2 times bigger than the corresponding values of the sub-grade, whereas in horizontal motion the stiffness of the sub-grade is about three orders of magnitude larger than that of the structure.

In case of the antenna, the static stiffness of the subgrade in vertical direction is about 4 times larger while in rotational direction about 2,5 times larger than the corresponding stiffness of the structure. For the same case, in horizontal direction the sub-grade stiffness may even be considered as rigid. In order to compare the numerical results it is of interest to compare the total masses of the structures. Both structures have a foundation block with a mass of $5,1 \times 10^{5} \mathrm{~kg}$. Without considering the foundation block the mass of the chimney is 17 times larger than the foundation mass, while the mass of the antenna is only $4,5 \%$ of the foundation mass.

\subsection{SSI Effects on Resonance Frequencies}

To check the numerical model, the first three eigenfrequencies for horizontal and vertical motion of the fixed beam, are calculated from the following equation

$$
\operatorname{det} K_{S S}^{S}=\left|K_{S S}^{S}\right|=0
$$

and compared with the analytical solutions for eigenfrequencies $\omega_{\mathrm{e}, n}$, where $n=1,2,3$ of the fixed beam [35]. Both solutions give the same results that are shown in Tab. 3 .

When dealing with free vibration problems, SEM leads to a transcendental eigenvalue problem, Eq. (15), with practically infinite number of eigenfrequencies. The problem is solved in this paper using MATLAB software [36]. It should be pointed out that FEM usually leads to a linear eigenvalue problem with finite number of eigenvalues that is equal to the number of DOF's of the system [37].

The eigenfrequencies of the fixed beam $\omega_{n}$ are equal to the values of $\omega$ for which the positive peaks of the following logarithmic function occur:

$$
f(\omega)=\log \frac{1}{\left|K_{S S}^{S}\left(\omega_{n}\right)\right|}
$$

The resonance frequencies $\omega_{\text {res, } n}$ of the coupled system (structure with soil) are calculated in the same way and compared with structural eigenfrequencies. The results are presented in Tab. 3 .

\begin{tabular}{|c|c|c|c|c|c|c|c|}
\hline & & \multicolumn{3}{|c|}{ Chimney } & \multicolumn{3}{|c|}{ Antenna } \\
\hline \multirow{2}{*}{ Direction } & \multirow{2}{*}{$\begin{array}{c}\mathrm{n} \\
\text { (mode) }\end{array}$} & w/o SSI & with SSI & freq. ratio & w/o SSI & with SSI & freq. ratio \\
\hline & & $\omega_{e, n}$ & $\omega_{\text {res }, n}$ & $\beta_{\text {res }, n}$ & $\omega_{e, n}$ & $\omega_{\text {res }, n}$ & $\beta_{\text {res }, n}$ \\
\hline \multirow{3}{*}{ Horizontal } & 1 & 1,49 & 0,57 & 0,38 & 25,56 & 19,5 & 0,76 \\
\hline & 2 & 9,32 & 6,41 & 4,30 & 167,6 & 160,2 & 6,27 \\
\hline & 3 & 26,11 & 18,85 & 12,66 & 456,4 & 448,6 & 17,55 \\
\hline \multirow{3}{*}{ Vertical } & 1 & 24,68 & 8,48 & 0,34 & 132,6 & 131,1 & 0,99 \\
\hline & 2 & 74,05 & 46,31 & 1,88 & 393,9 & 393,1 & 2,96 \\
\hline & 3 & 123,4 & 89,6 & 3,63 & 655,7 & 655,2 & 4,49 \\
\hline
\end{tabular}

Table 3 Eigenfrequencies and resonance frequencies for chimney and antenna

As expected, resonance frequencies of the chimney and of the antenna for horizontal and vertical motion, with SSI, are smaller, due to interaction effects, than the resonance frequencies calculated without SSI. In Tab. 3 , frequency ratio is defined as:

$\beta_{r e s, n}=\frac{\omega_{r e s, n}}{\omega_{e, n}}$

Coupling a much softer soil with a stiffer structure makes the whole system weaker and reduces the eigenvalues. This is specially emphasized in case of the chimney's horizontal motion due to the weak rotational stiffness of the foundation and in vertical motions due to the heavy mass of the structure. But, the resonant frequencies of the antenna's vertical motion with SSI are almost the same as in the case without SSI. It happens due to coupling of the stiffness of the beam with much larger stiffness of the soil in vertical direction, which makes the whole system behave as a fixed beam. Recent study [22] also showed that SSI affects more squat and stiff structures than the slender and tall ones.

\subsection{SSI Effects on Motion 4.2.1 Chimney}

Vertical excitation. The effect of SSI on vertical motion of the structure is shown in Figs. 5 and 6.

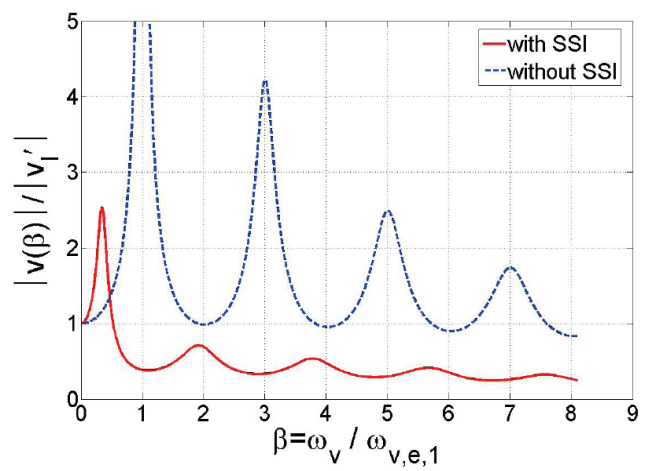

Figure 5 Frequency response for vertical excitation at top of chimney with SS and without SSI

In Fig. 5 the amplitude of vertical response at the top of the chimney $|v(\beta)|$ divided by the amplitude of input motion $\left|v_{\mathrm{I}}^{\prime}\right|$ is presented as a function of dimensionless frequency ratio:

$$
\beta=\frac{\omega_{v}}{\omega_{v, e, 1}}
$$


where $\omega_{v, e, 1}$ is the first eigenfrequency for vertical motion. With SSI the resonance frequencies are shifted to lower frequencies and the radiation damping reduces considerably the resonance amplitudes of the motion.

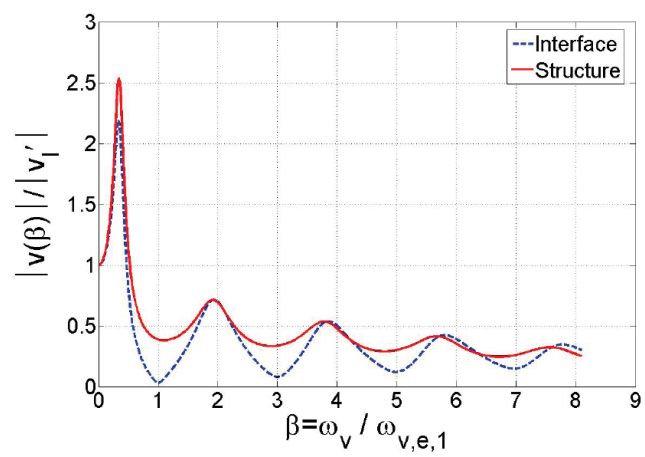

Figure 6 Frequency response for vertical excitation at top and base of chimney with SSI

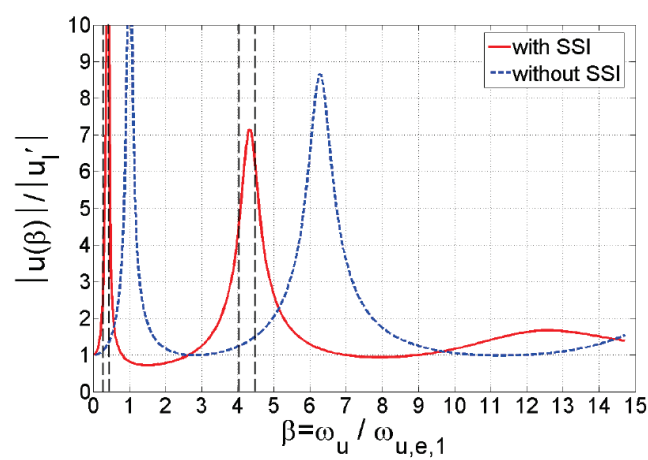

Figure 7 Frequency response for horizontal excitation at top of chimney with SSI and without SSI

The amplitudes of the base (interface) and the top of the chimney (structure) with SSI, normalized in the same way as for Fig. 5 are shown in Fig. 6. From the real and

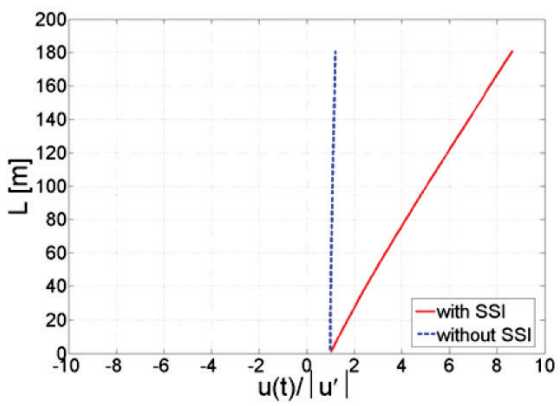

a) $\beta=0.35, \quad \omega=0.52$

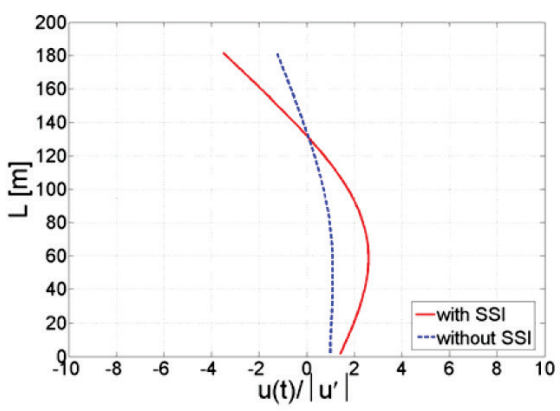

c) $\beta=4.0, \quad \omega=5.96$ imaginary (due to brevity not shown here) parts of the response we conclude that the top and the base of the structure at odd resonant frequencies vibrate in phase whereas at even resonance frequencies vibrate in opposite phase.

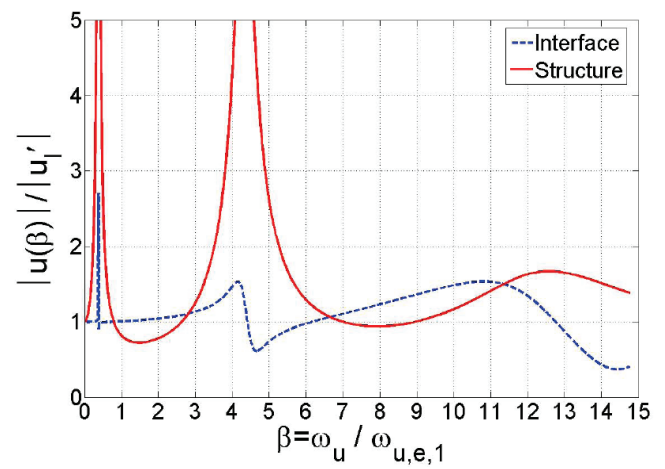

Figure 8 Frequency response for horizontal excitation at top and base of chimney with SSI

Horizontal excitation. Effects of SSI are shown in Figs. 7 and 8. In Fig. 7 the amplitude of horizontal response at the top of the chimney $|u(\beta)|$ divided by amplitude of input motion $\left|u_{I}^{\prime}\right|$ is presented as a function of dimensionless frequency

$$
\beta=\frac{\omega_{u}}{\omega_{u, e, 1}}
$$

where $\omega_{u, e, 1}$ is the first eigenfrequency for horizontal motion. The amplitudes of the base and the top of the chimney with SSI, normalized in the same way as for Fig. 7 are shown in Fig. 8.

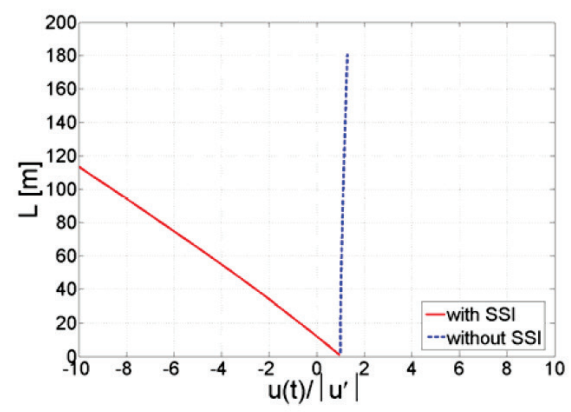

b) $\beta=0.4, \quad \omega=0.60$

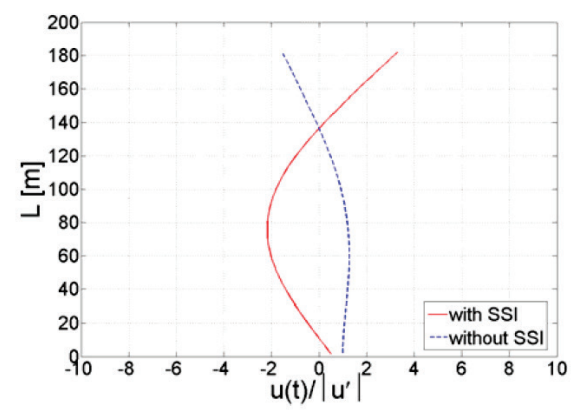

d) $\beta=4.5, \quad \omega=6.70$

Figure 9 Snapshots for horizontal motion of chimney at time $t=0, T, 2 T, \ldots$ at selected frequencies 
The snapshots show development of higher mode shapes with frequencies. Top and bottom move in phase below the first resonance frequency (Fig. 9a), whereas above the first resonance frequency they move out of phase (Fig. 9b). Near the second resonance frequency elastic deformation participates in the motion.

With SSI included resonance frequencies are shifted to lower frequencies but the radiation damping does not reduce $1^{\text {st }}$ resonance amplitude. The elasticity of the soil introduces a new low eigenform, see snapshots in Fig. 9. The essential motion is rotation of the base due to its large moment of inertia and the small radiation damping for rotation at low frequencies. Due to the large rigid body rotation the amplitudes at the case with SSI introduced are larger when compared to the case with no SSI (Fig. 9). At much higher $2^{\text {nd }}$ resonance frequency the increased rotational damping reduces the amplitude of the top of the structure. Snapshots of vibration forms at time $t=0, T$, $2 T, \ldots$, where $T=2 \pi \omega^{-1}$, are shown in Fig. 9. Those snapshots in Figs. 9a and $9 \mathrm{~b}$ are taken before and after the $1^{\text {st }}$ resonance frequency while the snapshots in Figs. 9c and $9 \mathrm{~d}$ are taken before and after the $2^{\text {nd }}$ resonance frequency. These frequencies are indicated by dashed vertical lines in Fig. 7.

\subsubsection{Antenna}

Vertical excitation. The effect of SSI can be seen in Tab. 3 and Figs. 10 and 11. Again, in Fig. 10 the amplitude of vertical response at the top of the antenna $|v(\beta)|$ divided by the amplitude of input motion $\left|v_{I}^{\prime}\right|$ is presented as a function of dimensionless frequency (17) in the same manner as in the case for chimney.

The amplitudes of the base and the top of the antenna with SSI, normalized in the same way as in Fig. 10, are shown in Fig. 11.

Since the sub-grade is very stiff with respect to the elasticity of the antenna, the resonance frequencies in cases with and without SSI effects included are almost the same. The radiation damping reduces the resonance amplitudes of the motion (Figs. 10 and 11). The rigid body motion of the structure at very low frequency $(\beta \approx$ 0,2 ) shows small resonance amplitude (Fig. 11). Large amplification at the top of the antenna occurs at the first eigenfrequency while amplitudes at higher resonance frequencies show strong damping. At higher frequencies the amplitudes of base plate become smaller and smaller.

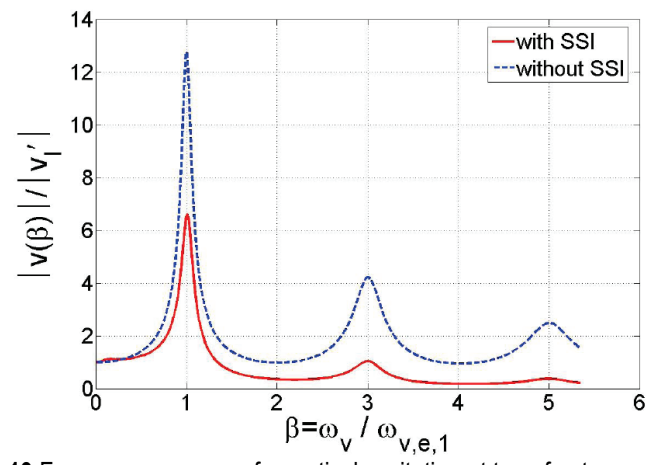

Figure 10 Frequency response for vertical excitation at top of antenna with SSI and without SSI

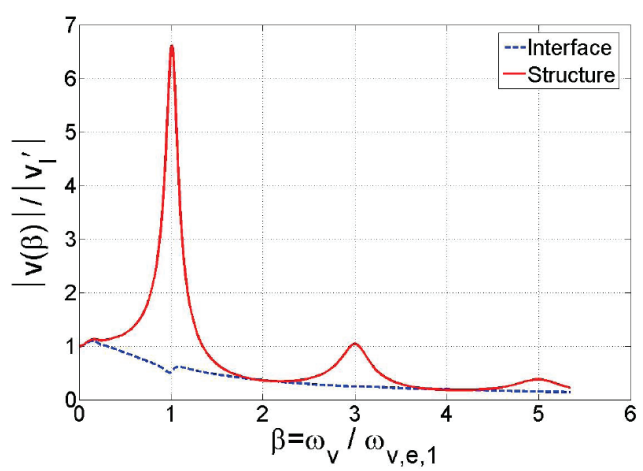

Figure 11 Frequency response for vertical excitation at top and base of antenna with SSI

Horizontal excitation. The response with and without SSI effects included have the same characteristics as for the vertical motion: the resonant frequencies are not shifted and the amplitudes are reduced; only the amplitude at first resonant frequency is larger due to rotation of the base (Tab. 3 and Figs. 12, 13).

Snapshots in Fig. 14 are taken at selected frequencies before and after the $1^{\text {st }}$ and the $2^{\text {nd }}$ resonance frequency, indicated by dashed vertical lines Fig. 12 .

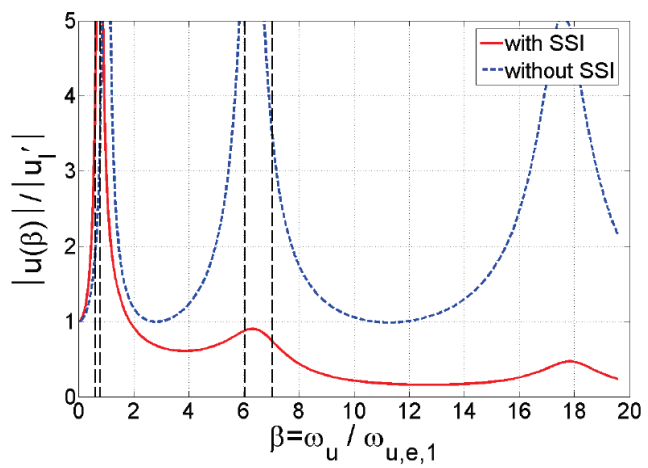

Figure 12 Frequency response for horizontal excitation at top of antenna with SSI and without SSI

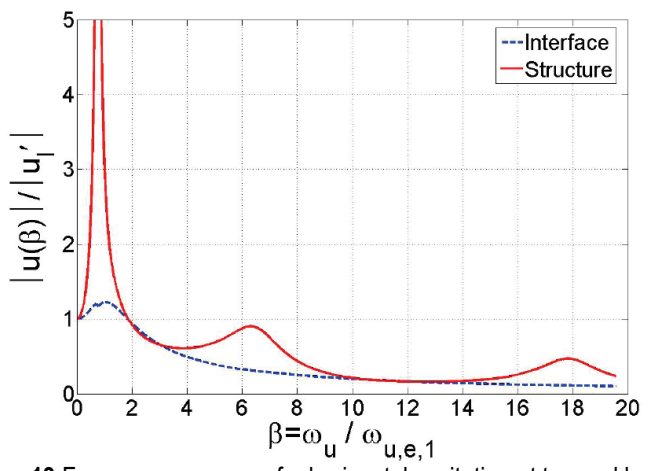

Figure 13 Frequency response for horizontal excitation at top and base of antenna with SSI

Those snapshots show the development of higher mode shapes with increasing frequencies. In Figs. 14a and $14 \mathrm{~b}$ the different mode shapes are shown before and after the first resonance respectively.

Before resonance the top and the base of the antenna move in phase while after resonance they move out of the phase. SSI affects the horizontal motion due to large base rotation. Namely, at higher frequencies the motion of the base is not so pronounced and it does not show the amplification of the free-field, which is just the opposite in the case of chimney (Figs. 13 and 8). The snapshots at 
higher frequencies show higher vibration mode shapes

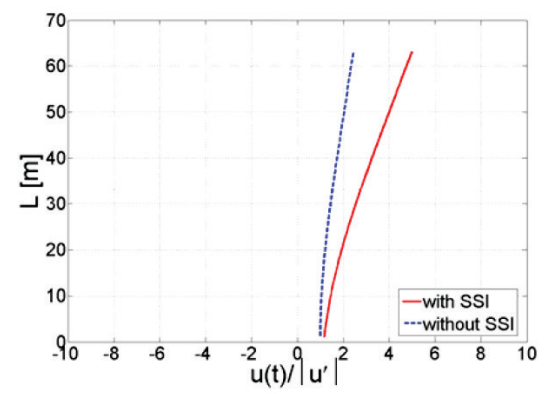

a) $\beta=0.7, \quad \omega=17.87$

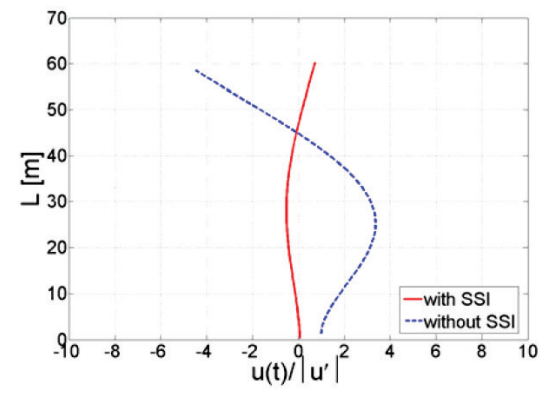

c) $\beta=6.0, \quad \omega=178.92$
(Figs. 14c and 14d).

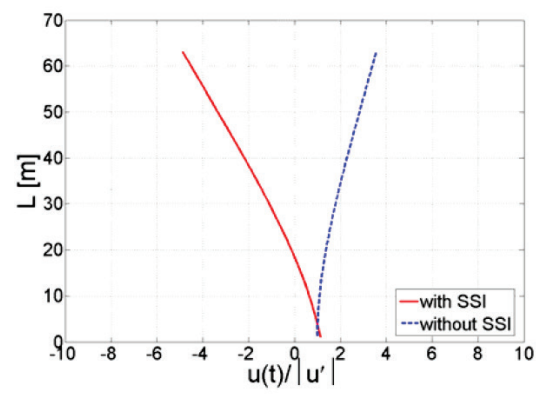

b) $\beta=0.8, \quad \omega=20.45$

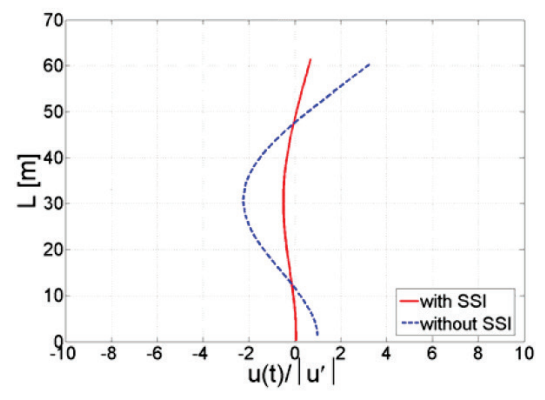

d) $\beta=7.0, \quad \omega=178.92$

Figure 14 Snap shots for horizontal motion of antenna at time $t=0, T, 2 T, \ldots$ at selected frequencies

\section{SUMMARY AND CONCLUSIONS}

If the dynamic interaction between the structure and sub-grade is formulated in total displacements (they include both kinematic and inertial interaction effects) the system response in the frequency domain is obtained as in the usual FE procedure. Analysis in the frequency domain has the advantage over the analysis in time domain since the numerical model can be solved without modelling errors. This is especially the case when a structure is modelled using spectral elements while the soil is modelled using analytical or semi-analytical procedures. Also, in comparison to the time domain, the calculation in frequency domain is much faster.

Two different structures, a chimney and an antenna, both modelled using one spectral Bernoulli beam element and founded on a shallow foundation of same geometry and weight were analyzed. Without considering the foundation block the mass of the chimney is 17 times larger than the foundation mass (heavy superstructure), while the mass of the antenna is only $4,5 \%$ of the foundation mass (light superstructure).

Material damping of the soil was set to zero in order to clearly show the influence of radiation damping on the structural response. Also, the coupling terms between swaying and rocking were neglected.

Static stiffness values for the chimney (stiff superstructure) for vertical and rotational motion are about 3,5 and 4,2 times bigger than the corresponding values of the sub-grade, whereas in horizontal motion the stiffness of the sub-grade is about three orders of magnitude larger than that of the structure. On the other hand, static stiffness of the sub-grade in vertical direction is about 4 times larger and in rotational direction about 2,5 times larger than the corresponding stiffness of the antenna (flexible superstructure). For that case, the sub- grade stiffness in horizontal direction may even be considered as rigid.

In the examples provided within this paper SSI effects are highlighted for vertical and horizontal excitation. Presented results show importance of the dynamic stiffness of the sub-grade. Also, they stress that different vibration forms create different damping effects through produced outgoing waves. The SSI analysis also shows the effect of the total mass on the base motion. Two structures having very different total masses produce different base motions.

The results obtained from analyses are summarized as follows:

1) For the heavy superstructure, resonance frequencies are shifted to lower values due to SSI effects for both, horizontal and vertical, excitations.

2) For the light superstructure, whose sub-grade is very stiff in comparison to the superstructure, resonance frequencies does not change due to SSI effects for both, horizontal and vertical, excitations.

3) In the case of vertical excitation, radiation damping reduces the amplitudes at the resonance frequencies for both cases, the stiff and the flexible superstructure.

4) For horizontal excitation, the top and the base for both, the stiff and the flexible, superstructures move in phase before resonance occurrence. After that they move out of phase.

5) For horizontal excitation, radiation damping does not reduce the amplitude of the 1 st resonance frequency for the stiff superstructure, while in the case of flexible superstructure the amplitude at the 1 st resonance frequency is reduced due to radiation damping.

6) In both cases, the stiff and the flexible structure, large base rotation occurs at horizontal excitation due to 
low radiation damping and large moment of inertia of the foundation.

The formulation provided in this paper can be extended and applied for general structures with an elastic foundation resting on layered sub-grade.

\section{ACKNOWLEDGEMENTS}

The German Johann Gottfried Herder Foundation Initiative made this international co-operation possible through the support of the guest professorship in Belgrade for the fourth mentioned author. Also, part of results reported here has been financed by the Ministry of Science of the Republic of Serbia through the Project TP 36046 and "FRAmed-MAsonry Composites for Modelling and Standardization" (HRZZ-IP-2013-113013) supported by the Croatian Science Foundation.

The authors are indebted to Professor Vladimir Sigmund (1956-2016) for his significant contribution and direction towards the research described in this paper.

\section{Appendix: Matrices of Interpolation Functions}

A) Axial deformation

Matrix of interpolation functions for axial deformation

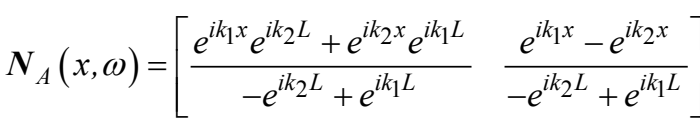

Wave numbers for axial deformation

$k_{1}=\omega \sqrt{\frac{\rho}{E}}, k_{2}=-\omega \sqrt{\frac{\rho}{E}}$

B) Bending

Matrix of interpolation functions for bending

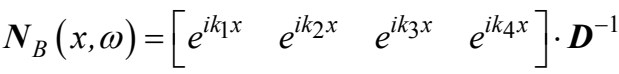

$\boldsymbol{D}=\left[\begin{array}{cccc}1 & 1 & 1 & 1 \\ i k_{1} & i k_{2} & i k_{3} & i k_{4} \\ e^{i k_{1} L} & e^{i k_{2} L} & e^{i k_{3} L} & e^{i k_{4} L} \\ i k_{1} e^{i k_{1} L} & i k_{1} e^{i k_{2} L} & i k_{1} e^{i k_{3} L} & i k_{1} e^{i k_{4} L}\end{array}\right]$

Wave numbers for bending

$k_{1}=\sqrt[4]{\frac{\rho A \omega^{2}}{E I}}, k_{2}=i \sqrt[4]{\frac{\rho A \omega^{2}}{E I}}$,

$k_{3}=-\sqrt[4]{\frac{\rho A \omega^{2}}{E I}}$ and $k_{4}=-i \sqrt[4]{\frac{\rho A \omega^{2}}{E I}}$
6 REFERENCES

[1] CEN (Comité Européen de Normalisation), (2004). Eurocode 8: Design of structures for earthquake resistance, Part 5: Foundations, retaining structures and geotechnical aspects, EN 1998-5. Brussels, Belgium

[2] CEN (Comité Européen de Normalisation), (2005). Eurocode 8: Design of structures for earthquake resistance, Part 6: Towers, masts and chimneys, EN 1998-6. Brussels, Belgium

[3] Siefert, J. \& Cevaer, F. (1992). Handbook of Impedance Functions - Surface Foundations. Press Académiques, Nantes.

[4] Hillmer, P. \& Schmid, G. (1988). Analysis of a dynamically loaded frame structures with local nonlinearities using Laplace transforms. Ingenieur-Archiv, 58, 148-160. https://doi.org/10.1007/BF00536234

[5] Hillmer, P. \& Schmid, G. (1988). Calculation of foundation uplift effects using a numerical Laplace transform. Earthquake Engineering \& Structural Dynamics, 16, 789 801. https://doi.org/10.1002/eqe.4290160602

[6] Kolousek, V. (1941). Anwendung des Gesetzes der virtuellen Verschiebungen und des Reziprozitätssatzes in der Stabwerksdynamik. Ingenieur-Archiv, 12, 363-370. https://doi.org/10.1007/BF02089894

[7] Kolousek, V. (1973). Dynamics in Engineering Structures. Butterworth, Londin.

[8] Clough, R. \& Penzien, J. (1993). Dynamcs of Structures. McGraw-Hill, Singapore.

[9] Banerjee, J. \& Williams, F. (2003). Coupled bendingtorsional dynamic stiffness matrix of an axially loaded Timoshenko beam by spectral element method. Journal of Sound and Vibration, 264, 1139-1153. https://doi.org/10.1016/S0022-460X(02)01387-1

[10] Krawszuk, M., Palcz, M., \& Ostachowicz, W. (2007). The dynamic analysis of a cracked Timoshenko beam by the spectral element method. Journal of Sound and Vibration, 304. 124-153. https://doi.org/10.1016/j.jsv.2007.02.013

[11] Viola, U., Ricci, P., \& Aliabadi, M. (2007). Free vibration analysis of axially loaded Timoshenko beam structure using dynamic stiffness method. Journal of Sound and Vibration, 304, 124-153. https://doi.org/10.1016/j.jsv.2007.02.013

[12] Doyle, J. (1997). Wave Propagation in Structures: Spectral Analysis Using Fast Discrete Fourier Transform. SpringerVerlag, New York Inc. https://doi.org/10.1007/978-1-4612-1832-6

[13] Spyrakos, C. \& Beskos, D. (1982). Dynamic response of frameworks by Fast Fourier Transform. Computer \& Structures, 15, 495-505. https://doi.org/10.1016/0045-7949(82)90001-3

[14] Igawa, H., Komatsub, K., Yamaguchia, I., \& Kasai, T. (2004). Wave propagation analysis of frame structures using the spectral element method. Journal of Sound and Vibration, 277, 1071-1081. https://doi.org/10.1016/j.jsv.2003.11.026

[15] Penava, D. (2008). Vibration analysis of frame structures using spectral element method. MSc thesis. Faculty of Civil Engineering in Belgrade, Serbia.

[16] Penava, D., Schmid, G., Sigmund, V., \& Petronijević, M. (2008). Vibration analysis of frame structures excided with Rayleigh waves using spectral elements. International Scientific Symposium Modeling of Structures / Mostar, BiH, 545-558.

[17] Penava, D., Bajrami, N., Schmid, G., Petronijević, M., Aleksovski, G., \& Sigmund, V. (2008). Dynamic SoilStructure Interaction of frame structures with spectral elements. Part II. Proceddings of the $2^{\text {nd }}$ International Scientific Conference / Podgorica, Montenegro, 109-114 
[18] Petronijević, M., Nefovska-Danilovič, M., \& Radišić, M. (2013). Analysis of Frame Structure Vibrations Induced by Traffic. Gradevinar, 65, 814-825.

[19] Dominguez, J. (1993). Boundary Elements in Dynamics. Computational Mechanic Publications, Southampton, Boston.

[20] Kausel, E. (1994). Thin-Layer method. International Journal for Numerical Methods in Engineering, 37, 927941. https://doi.org/10.1002/nme.1620370604

[21] Kalinchuk, V., Belyankova, T., \& Tosecký, A. (2005). The effective approach to the in-homogeneous media dynamics modelling. Eurodyn 2005 / Millpress, Rotterdam, 21992204.

[22] Kraus, I. (2014). Seismic response spectra of shallow founded structures on soft soils. PhD thesis. University of Osijek, Faculty of Civil Engineering Osijek, Osijek, Croatia.

[23] Jennings, P. C. \& Bielak, J. (1972). Dynamics of buildingsoil interaction. Report No. EERL 72-01. Earthquake Engineering Research Laboratory, California Institute of Technology, Pasadena, California, USA.

[24] Gazetas, G. (1983). Analysis of machine foundation vibrations: state of the art. International Journal of Soil Dynamics and Earthquake Engineering, 2(1), 2-42. https://doi.org/10.1016/0261-7277(83)90025-6

[25] Çelebi, E., Furat, S., \& Çankaya, İ. (2006). The evaluation of impedance functions in the analysis of foundation vibrations using boundary element method. Applied Mathematics and Computation, 173(1), 636-667. https://doi.org/10.1016/j.amc.2005.04.006

[26] Pitilakis, D. \& Clouteau, D. (2010). Equivalent linear substructure approximation of soil-foundation-structure interaction: model presentation and validation. Bulletin of Earthquake Engineering, 8(2), 257-282. https://doi.org/10.1007/s10518-009-9128-3

[27] Lee, U., Kim, J., \& Leung, A. (2000). The spectral element method in structural dynamics. The Schock and Vibration Digest, 32, 451-465. https://doi.org/10.1177/058310240003200601

[28] Veletsos, A. S. \& Verbič, B. (1973). Vibration of viscoelastic foundations. Report No. 18. Department of Civil Engineering, Rice University, Houston, Texas, USA. https://doi.org/10.1002/eqe.4290020108

[29] Wolf, J. P. (1985). Dynamic soil-structure interaction. Prentice-Hall, Inc., Englewood Cliffs, New Jersey, USA

[30] Kramer, S.L. Geotechnical earthquake engineering. Prentice Hall, Upper Saddle River, New Jersey, USA, 1996.

[31] Ordóñez, G. A. (2011). SHAKE2000 - A computer program for the 1-D analysis of geotechnical earthquake engineering problems: user's manual. GeoMotions, LLC, Lacey, Washington, USA.

[32] Friedrich, K. \& Tosecký, A. (2003). Impedanzfunktionen rechteckiger Fundamente - Interner Bericht. Theorie der Tragwerke und Simulationstechnik, Ruhr-Universität Bochum.

[33] Tabatabaie, M., Mejia, L., Wu, S. C., \& Galagoda, M. (2004). Dynamic stiffness for vibration analysis of large machine foundations on soft soils stabilized by deep mixing. The $11^{\text {th }}$ International Conference on Soil Dynamics \& Earthquake Engineering ( $11^{\text {th }}$ ICSDEE) and the $3^{\text {rd }}$ International Conference on Earthquake Geotechnical Engineering ( $\left.3^{\text {rd }} I C E G E\right)$ / University of California, Berkeley, 782-789

[34] Pitilakis, D., Moderessi-Farahmand-Razavi, A., \& Clouteau, D. (2013). Equivalent-linear dynamic impedance functions of surface foundations. Journal of Geotechnical and Geoenvironmental Engineering, 139(7), 1130-1139. https://doi.org/10.1061/(ASCE)GT.1943-5606.0000829
[35] Kanovsky, J. A. \& Lebed, O. I. (2000). Formulas for Structural Dynamics - Tables, Graphs and Solutions. McGraw-Hill.

[36] MATLAB 2011. (2011). The Language of Technical Computing. MathWorks Inc.

[37] Kolekova, Y., Petronijević, M., \& Schmid, G. (2010). Special Dynamic Soil-Structure Analysis Procedures Demonstrated for Tower-Like Structures. Slovak Journal of Civil Engineering, 18(2), 26-33.

\section{Contact information:}

Davorin PENAVA, PhD, Assistant Professor

J. J. Strossmayer University of Osijek

Faculty of Civil Engineering Osijek

Vladimira Preloga 3, 31000 Osijek, Croatia

dpenava@gfos.hr

Ivan KRAUS, PhD, Assistant Professor

J. J. Strossmayer University of Osijek

Faculty of Civil Engineering Osijek

Vladimira Preloga 3, 31000 Osijek, Croatia

ikraus@gfos.h

Mira PETRONIJEVIĆ, PhD, Full Professor

University of Belgrade

Faculty of Civil Engineering

Bulevar kralja Aleksandra 73, 11000 Beograd, Serbia

pmira@grf.bg.ac.rs

Günther SCHMID, PhD, Full Professor

Ruhr-Universität Bochum

Fakultät für Bau- und Umweltingenieurwissenschaften

Universitätsstraße 150, 44801 Bochum, Germany

guenther.schmid@ruhr-uni-bochum.de 